\title{
Community-Centric Graph Convolutional Network for Unsupervised Community Detection
}

\author{
Dongxiao He ${ }^{1}$, Yue Song ${ }^{1}$, Di Jin ${ }^{1, *}$, Zhiyong Feng ${ }^{1}$, \\ Binbin Zhang ${ }^{1}$, Zhizhi Yu ${ }^{1}$ and Weixiong Zhang ${ }^{2}$ \\ ${ }^{1}$ College of Intelligence and Computing, Tianjin University, Tianjin 300350, China \\ ${ }^{2}$ Department of Computer Science and Engineering, Washington University, St. Louis, MO 63130, USA \\ \{hedongxiao, sy717389667, jindi, zyfeng\}@tju.edu.cn, weixiong.zhang@wustl.edu
}

\begin{abstract}
Community detection, aiming at partitioning a network into multiple substructures, is practically importance. Graph convolutional network (GCN), a new deep-learning technique, has recently been developed for community detection. Markov Random Fields (MRF) has been combined with GCN in the MRFasGCN method to improve accuracy. However, the existing GCN community-finding methods are semi-supervised, even though community finding is essentially an unsupervised learning problem. We developed a new GCN approach for unsupervised community detection under the framework of Autoencoder. We cast MRFasGCN as an encoder and then derived node community membership in the hidden layer of the encoder. We introduced a community-centric dual decoder to reconstruct network structures and node attributes separately in an unsupervised fashion, for faithful community detection in the input space. We designed a scheme of local enhancement to accommodate nodes to have more common neighbors and similar attributes with similar community memberships. Experimental results on real networks showed that our new method outperformed the best existing methods, showing the effectiveness of the novel decoding mechanism for generating links and attributes together over the commonly used methods for reconstructing links alone.
\end{abstract}

\section{Introduction}

Real-world systems often appear in the form of networks. Examples include social networks, power grid and world trade networks. Real networks have modular structures or communities [Fortunato, 2010], which are densely connected subgraphs with nodes of close relationships and similar properties. Identification of network modular structures is an effective means to understanding the underlying organizational principles and functions of the system that the network represents. Community detection has been an active area of research, as surveyed in [Falih et al., 2018; Fortunato, 2010;

\footnotetext{
${ }^{*}$ Corresponding author.
}

Fortunato and Hric, 2016], and many community detection methods have been proposed, including that based on statistical modeling [Chen et al., 2018], modularity optimization [Yang et al., 2016], matrix factorization [Wang et al., 2017].

Deep learning has recently been adopted in network analysis [Kipf and Welling, 2017; Yang et al., 2016; Jin et al., 2018; Monti et al., 2018; Pan et al., 2018]. In particular, Graph Convolutional Networks (GCN) has attracted a great deal of attention lately due to its success on supervised and semi-supervised classification of nodes in a graph [Li et al., 2018; Kipf and Welling, 2017] which can be adopted for community detection. Of particular relevance to the current study is MRFasGCN [Jin et al., 2019], a state-of-the-art GCNbased semi-supervised community detection method, which incorporates a Markov Random Fields (MRF) modeling of communities in the GCN framework.

Community detection is in essence an unsupervised learning problem. Real-world networks are typically unique - the training data from one network can be hardly used adequately for another network. As a corollary to this observation, when finding communities in a network, the only data available for analysis are the information on the network itself. A scheme of semi-supervised learning, such as the MRFasGCN method, can apply in such a way that partial label information on some of the nodes in a given network can be used to predict the community identities of the remaining unlabeled nodes in the same network. However, for most application problems, even such partial training data are costly and arduous to gather.

Therefore, to advance the state-of-the-art of community detection, it is of great technical significance and paramount practical importance to develop GCN-based algorithm for unsupervised community detection by exploiting the great power of automatic feature learning and effective optimization that deep learning can offer. It also remains to be seen if an end-to-end deep learning approach can outperform the existing methods that use statistical methods and other machine learning techniques for community finding.

We developed a novel GCN-based approach for Unsupervised Community Detection in attribute networks, referred to as GUCD. In this method, we incorporated network modeling method of MRFasGCN in the Autoencoder framework and introduced a special neural network architecture suitable for learning network communities and node semantics at the same time. We further introduced a local enhancement to the 
latent communities, i.e. we first constructed an aggregated graph combining the information of both topology and attributes, and then made each pair of connected nodes in this graph have similar community distributions. We conducted experiments on some real networks to compare the new approach with the best existing methods and analyzed the features of the new approach. To our best knowledge, GUCD is the first GCN method for unsupervised community finding.

\section{Preliminaries}

We first introduce some notations and define the problem of community detection, and then discuss MRFasGCN [Jin et al., 2019] (a GCN based semi-supervised community detection method) which serve as the bases of our new approach.

\subsection{Notations and Problem Definition}

An undirected and attributed network is represented as a graph $G=(V, E, W)$ over a set of $n$ nodes $V=$ $\left\{v_{1}, v_{2}, \ldots, v_{n}\right\}$, a set of $e$ edges $E$ with $e_{i j}=\left(v_{i}, v_{j}\right) \in E$ if an edge exists between nodes $v_{i}$ and $v_{j}$, and a set of $m$ node attributes $W=\left\{w_{1}, \ldots w_{m}\right\}$. The topological structure of $G$ is represented by an $n \times n$ adjacency matrix $A=\left(a_{i j}\right)_{n \times n}$, where $a_{i j}=1$ if $e_{i j} \in E$, or 0 otherwise. An $n \times m$ attribute matrix $X$ is used to denote the attributes of nodes, where $x_{i t}=1$ if a node has attribute $w_{t}$, or 0 otherwise.

Given an attribute network $G$, community detection is to partition the $n$ nodes into $K$ communities $C=$ $\left\{C_{1}, C_{2}, \ldots, C_{K}\right\}$ so that each node $v_{i}$ has a community identity or label $c_{i} \in L=\{1,2, \ldots, K\}$.

\subsection{MRFasGCN}

MRFasGCN [Jin et al., 2019] infers a partition of nodes in a network in two main parts. First, they take the original two convolutional layers of GCN [Kipf and Welling, 2017], that is

$$
X^{(2)}=\operatorname{softmax}\left(\hat{A} \operatorname{ReLU}\left(\hat{A} X H^{(0)}\right) H^{(1)}\right)
$$

as the first two layers of the neural network to infer an initial assignment of node labels $\left(X^{(2)}\right)$, where $\hat{A}=$ $\tilde{D}^{-1 / 2} \tilde{A} \tilde{D}^{-1 / 2}\left(\tilde{A}=A+I_{n}\right.$ and $\tilde{D}$ is a diagonal matrix with $\tilde{d}_{i i}=\sum_{j} \tilde{a}_{i j}$ ) captures network topology, $X$ is the attribute matrix, and $H^{(0)}$ and $H^{(1)}$ are weight parameters of the two convolutional layers to be trained.

MRFasGCN then incorporates a community-oriented MRF as the third convolutional layer in the GCN. The central piece of this MRF model is an energy function, $E(C \mid A, X)$, with two parts: the sum of unary potentials over all nodes and the sum of pairwise potentials over all edges:

$$
\begin{aligned}
E(C \mid A, X) & =\alpha * \sum_{i} \phi_{u}\left(c_{i}\right)+(1-\alpha) * \sum_{i \neq j} \phi_{p}\left(c_{i}, c_{j}\right) \\
& =\alpha * \sum_{i}-p\left(c_{i}\right)+(1-\alpha) * \sum_{i \neq j} \mu\left(c_{i}, c_{j}\right) \tau\left(v_{i}, v_{j}\right)
\end{aligned}
$$

where $\alpha$ is a parameter for balancing the unary and pairwise potentials. The unary potential $\phi_{u}\left(c_{i}\right)=-p\left(c_{i}\right)$ measures the cost for node $v_{i}$ taking label $c_{i}$, where $p\left(c_{i}\right)=x_{i, c_{i}}^{(2)}$ is the probability that $v_{i}$ has label $c_{i}$, derived from GCN in Eq. (1). The pairwise potential $\phi_{p}\left(c_{i}, c_{j}\right)=\mu\left(c_{i}, c_{j}\right) \tau\left(v_{i}, v_{j}\right)$ measures the cost for assigning labels $c_{i}$ and $c_{j}$ to nodes $v_{i}$ and $v_{j}$, where $\mu\left(c_{i}, c_{j}\right)$ denotes the semantic similarities between communities $c_{i}$ and $c_{j}$, and $\tau\left(v_{i}, v_{j}\right)$ the attribute similarity or consistency between nodes $v_{i}$ and $v_{j}$, defined as:

$$
\begin{gathered}
\mu\left(c_{i}, c_{j}\right)=(-1)^{\delta\left(c_{i}, c_{j}\right)} h_{c_{i} c_{j}}^{(2)} \\
\tau\left(v_{i}, v_{j}\right)=\beta * \xi\left(v_{i}, v_{j}\right)+(1-\beta) * R_{i}\left(\zeta\left(v_{i}, v_{j}\right)\right)
\end{gathered}
$$

where $h_{c_{i} c_{j}}^{(2)}$ is the parameter to be learned, $\delta\left(c_{i}, c_{j}\right)=1$ if $c_{i}=c_{j}$, or 0 otherwise, $\xi\left(v_{i}, v_{j}\right)=d_{i} d_{j} / 2 e-a_{i j}$ ( $d_{i}$ is the degree of node $v_{i}$ and $e$ the number of edges), and $\beta$ is a tradeoff parameter that balances topology and attributes. $\zeta\left(v_{i}, v_{j}\right)$ is defined by using the cosine similarity between the attribute vectors of nodes $v_{i}$ and $v_{j}$, and then an asymmetric regularization is used to balance the difference of the sum of similarity on every node, i.e., $R_{i}\left(\zeta\left(v_{i}, v_{j}\right)\right)=$ $\zeta\left(v_{i}, v_{j}\right) / \sum_{t=1}^{n} \zeta\left(v_{i}, v_{t}\right)$.

However, minimizing the above energy function in order to yield the most probable community partition for a given network is intractable, since the pairwise potentials are defined over a complete graph rather than the sparse network. Thus, a mean filed approximation is adopted to approximate the exact distribution $P(C \mid A, X)$. The updating procedure for this approximation has four steps 1) initialization, 2) message passing, 3) adding unary potentials and 4) normalization. Following these steps, MRFasGCN can transform the MRF's inference into a layer of convolutional process (that is compatible with GCN of Eq. (1)), defined as:

$$
Z=\operatorname{softmax}\left(X^{(2)}-\Upsilon X^{(2)} H^{(2)}\right)
$$

where $\Upsilon=\left(\tau\left(v_{i}, v_{j}\right)\right)_{n \times n}$ is defined in Eq. (4), $H^{(2)}$ are the weight parameters to be trained, and $Z=\left(z_{i c_{i}}\right)_{n \times K}$ the final community membership of nodes (where $z_{i c_{i}}$ denotes the probability that node $v_{i}$ belongs to community $c_{i}$ ).

\section{The Method}

After a brief overview of our new method, we will introduce an encoder based on MRFasGCN to derive node community memberships and a dual decoder for reconstructing links and attributes based on communities. We then discuss a regularization to enhance community detection locally.

\subsection{Overview}

The new GCN-based approach for Unsupervised Community Detection, short-handed as GUCD, adopts Autoencoder as its overall architecture and includes three main parts (Fig. 1). In the first part (the green box to the left of Fig. 1), we adopted the three convolutional layers of MRFasGCN as the encoder of GUCD, where the first two layers were to learn a deep representation of the attribute network and the third layer was to model and derive node community membership using both the deep representation and network information. We then designed a dual decoder as the second part of GUCD (the red box to the right of Fig. 1), using the derived communities to separately reconstruct network topology and node attributes. 


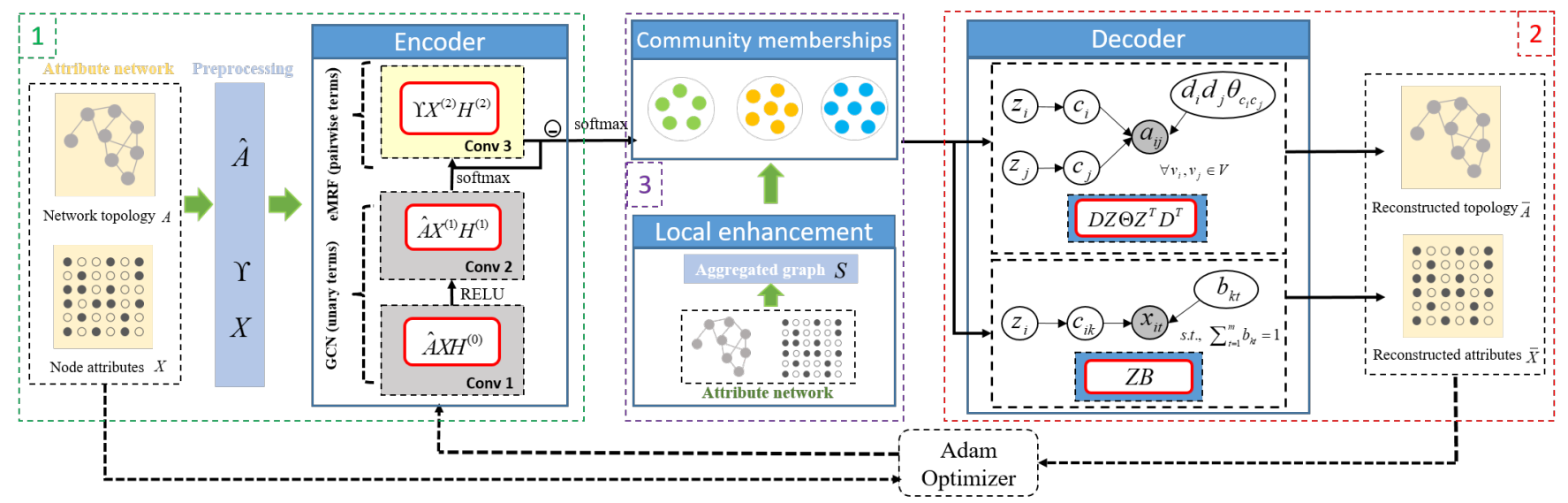

Figure 1: The architecture of GUCD. Part 1 to the left in the green box represents the encoder for deriving community membership of nodes. Part 2 to the right in the red box represents the dual decoder for reconstructing links and node attributes using community membership. Part 3 in the middle in the purple box represents the local enhancement on communities.

We first reconstructed the network topology by requiring the nodes within the same community to maintain the same link pattern (with also considering the heterogeneity of node degrees) connecting to the rest of the network, which is suitable for generating the coupling relationships between nodes. We then generated node contents using topic modelling, i.e. we assumed that the contents of nodes in the same community share similar distributions of attribute words used. We formulated the dual decoder to fully utilize (structural and content) data from diverse sources, making the decoding process suitable for unsupervised community detection. In the third part (in purple box in the middle of Fig. 1), we added a regularization of local enhancement to the latent communities, i.e. we first constructed an aggregated graph combining the information of both topology and attributes, and then made each pair of connected nodes in this graph have similar community distributions. The model was trained as a whole using the Adam optimizer [Kingma and $\mathrm{Ba}, 2015$ ].

\subsection{The Shared Encoder}

We processed the network topology and node attributes together using the same encoder so as to extract hidden network characteristics, particularly latent community structures to be identified. To be specific, we adopted the three convolutional layers of MRFasGCN [Jin et al., 2019] as the encoder (the green box of Fig. 1). We used the first two layers to derive a general embedding and then derived an initial node community labels $X^{(2)}$ (defined in Eq. (1)) by using softmax on the embedding, which is not community-specific. We used this initial solution $X^{(2)}$ to define unary potentials in MRF, and then defined pairwise potentials of MRF to model communities $Z$ (defined in Eq. (5)) hidden in network topology and attributes, leading to the third convolutional layer. Through these three layers, the derived node community labels $Z$ not only utilize the deep representation but also are smoothly community-oriented. The node to community assignments in $Z$ will be learned when the whole model with encoder and decoder is trained together; the high quality of the reconstructed network will require an accurate commu- nity structure at the end of the encoder.

\subsection{The Dual Decoder}

The dual decoder is the core part of the new method. It consists of two decoders, one for reconstructing network topology and the other for reconstructing node attributes.

\section{The Decoder for Reconstructing Network Topology}

This novel decoder attempts to reconstruct network topology based on the node community membership derived in the latent space. It not only makes the decoder communityoriented, but also achieves unsupervised learning for community detection. The idea is inspired by the block model for blocks, groups, or communities in networks. That is, if the community which a node $v_{i}$ belongs to is denoted as $c_{i}$ $\left(c_{i}=1,2, \ldots, K\right)$, we can then define a $K \times K$ block matrix $\Theta$ such that each element $\theta_{r s}$ in the matrix is the possibility of having an edge between any two nodes $v_{i}$ and $v_{j}$ (with $c_{i}=r$ and $c_{j}=s$ ). In this case, the nodes in each of the $K$ communities (with label $r$ ) have the same link pattern, i.e., the nodes in the same community share the same link probability with any node $v_{j}$ in the network, i.e. $\theta_{r c_{j}}$. This idea naturally describes the coupling relationship between nodes in the network since it is defined to generate pairwise rather than individuals based on community structure. The model is further improved by considering the heterogeneity of node degrees, i.e. the nodes with higher degrees should be more likely to be connected. Therefore, the model can be revised such that the possibility that nodes $v_{i}$ and $v_{j}$ are connected is $d_{i} d_{j} \theta_{c_{i} c_{j}}$, where $d_{i}$ is the degree of $v_{i}$. This mechanism is in concordance with the degree-corrected stochastic block model (DCSBM) [Karrer and Newman, 2011] even though the formulation is different.

Based on the above model, the expected number of links between nodes $v_{i}$ and $v_{j}$, which respectively belong to communities $c_{i}$ and $c_{j}$, can be written as

$$
\bar{a}_{i j}^{c_{i}, c_{j}}=\operatorname{sigmoid}\left(z_{i c_{i}} z_{j c_{j}} d_{i} d_{j} \theta_{c_{i} c_{j}}\right)
$$

where $z_{i c_{i}}$ is the probability that $v_{i}$ belongs to community $c_{i}$, which is from the encoder, defined in Eq. (5). Considering 
all communities $\left\{\left(c_{i}, c_{j}\right) \mid c_{i}, c_{j} \in L\right\}$, we can then define the expected number of links between nodes $v_{i}$ and $v_{j}$ as:

$$
\bar{a}_{i j}=\operatorname{sigmoid}\left(\sum_{c_{i}=1}^{K} \sum_{c_{j}=1}^{K} z_{i c_{i}} z_{j c_{j}} d_{i} d_{j} \theta_{c_{i} c_{j}}\right)
$$

By considering both the block modelling and heterogeneity degree of nodes, the model can well describe the coupling relationship among nodes in the network with community structures. The above model can be formulated as a layer of a neural network in order to incorporate it into the Autoencoder framework. As the $K$-dimensional vector $\vec{z}_{i}=\left(z_{i c_{i}}\right)_{1 \times K}$ denotes the probability distribution of $v_{i}$ belonging to different communities, the link propensity between nodes $v_{i}$ and $v_{j}$ can be revised to $\bar{a}_{i, j}=\operatorname{sigmoid}\left(d_{i}\left(\vec{z}_{i} \Theta \vec{z}_{j}^{T}\right) d_{j}\right)$, which can also be written in a matrix form as:

$$
\bar{A}=\operatorname{sigmoid}\left(D Z \Theta Z^{T} D^{T}\right)
$$

where $D=\operatorname{diag}\left(d_{1}, d_{2}, \cdots, d_{n}\right)$. Taking $\Theta$ as the weight parameters of the neural network, Eq. (8) can then be represented by a layer of the neural network, i.e., the topological decoder. The parameters $\Theta$ can be learned by minimizing the difference between the observed adjacency matrix $A$ and the adjacency matrix $\bar{A}$ generated from the model. This difference can be defined by using the cross-entropy loss as:

$$
L_{\text {topo }}=-\sum_{i, j=1}^{n}\left[a_{i j} \ln \bar{a}_{i j}+\left(1-a_{i j}\right) \ln \left(1-\bar{a}_{i j}\right)\right]
$$

\section{The Decoder for Reconstructing Attributes}

Reconstruction of node attributes stems in topic modelling [Blei et al., 2003]. Nodes with similar semantic attributes are believed to be more likely to belong to the same community. Thus, the nodes in the same community are more likely to have similar distributions of attribute words, and different communities in a network can be characterized as representing different semantic topics.

Let $P\left(c_{i} \mid v_{i}\right)=z_{i c_{i}}$ be the probability that node $v_{i}$ belongs to community $c_{i}$, where $Z=\left(z_{i c_{i}}\right)_{n \times K}$ is the matrix of node community memberships from the encoder; and $P\left(x_{i t}=1 \mid c_{i}=k\right)=b_{k t}$ the probability that the $k$ th $\left(c_{i}=k\right)$ community/topic selects an attribute word $w_{t}$ from the entire word set $w_{t} \in W$, where $B=\left(b_{k t}\right)_{K \times m}$ is to be learned. Assume that every node-attribute pair $\left\langle v_{i}, w_{t}\right\rangle$ in the context (with $x_{i t}=1$ ) is generated independently. Then, given the community $c_{i}$ of node $v_{i}$, the probability for a node-attribute pair $\left\langle v_{i}, w_{t}\right\rangle$ generated will be:

$$
\bar{x}_{i t}^{c_{i}}=P\left(x_{i t}=1 \mid c_{i}\right) P\left(c_{i} \mid v_{i}\right)
$$

Considering all communities, the probability that $\left\langle v_{i}, w_{t}\right\rangle$ appears in the context will be:

$$
\bar{x}_{i t}=P\left(x_{t} \mid v_{i}\right)=\sum_{c_{i} \in L} P\left(x_{i t}=1 \mid c_{i}\right) P\left(c_{i} \mid v_{i}\right)
$$

The above generative process can be formulated as a layer of neural network to serve as an attributed decoder. Using
$\bar{X}=\left(\bar{x}_{i t}\right)_{n \times m}$, the matrix form of the above model is:

$$
\bar{X}=Z \cdot B \quad \text { s.t. }, \quad \sum_{t=1}^{m} b_{k t}=1
$$

where $B$ denotes the weight parameters in this neural network layer to be trained. Then, the likelihood that the attribute matrix $X$ is generated by the above model is:

$$
\begin{aligned}
P(X \mid V) & =\prod_{i=1}^{n} \prod_{t=1}^{m} \sum_{c_{i} \in L} P\left(x_{i t}=1 \mid c_{i}\right) P\left(c_{i} \mid v_{i}\right) \\
& =\prod_{i=1}^{n} \prod_{t=1}^{m}\left(\sum_{c_{i} \in L} z_{i c_{i}} b_{c_{i} t}\right)^{x_{i t}}=\prod_{i=1}^{n} \prod^{m}\left(\bar{x}_{i t}\right)^{x_{i t}}
\end{aligned}
$$

where $x_{i t}=1$ if node $v_{i}\left(v_{i} \in V\right)$ has attribute $w_{t}$, or 0 otherwise. Since the objective of the network generative process is to maximize this likelihood, the negative log-likelihood is then taken as the loss of this layer of the neural network,

$$
L_{\text {attr }}=-\sum_{i=1}^{n} \sum_{t=1}^{m} x_{i t} \ln \bar{x}_{i t}
$$

\subsection{Local Enhancement}

Different from the main model (e.g. the MRF part) which describes communities via global information, we further utilize local information to enhance communities from a local view. The rationale is that two nodes should have similar node community membership if they are close to each other, topologically and/or semantically, in the attribute network.

We implemented this local enhancement scheme by introducing pairwise constraints on nodes and a graph regularization term to the objective function. We first built an aggregated graph based on network topology and node attributes. To measure the proximity of a pair of nodes, we introduced a measurement method to combine their topological and attribute similarities. We computed the set of the local neighbors for each node $v_{i}$, i.e.,

$$
\Gamma_{i}=\Gamma_{i}^{t o p o} \cup \Gamma_{i}^{a t t r}
$$

where $\Gamma_{i}^{t o p o}$ is the set of neighbors directly adjacent to $v_{i}$, and $\Gamma_{i}^{a t t r}$ the set of top- $k_{a t t r}$ most attribute-similar neighbors of $v_{i}$, where the TF-IDF cosine similarity is used to calculate the attribute similarity of nodes. That is, let $\vec{x}_{i}$ be the attribute vector of node $v_{i}$, then for attribute unit $x_{i t}$, its TF-IDF value in a term vector $\vec{x}_{i}$ is:

$$
\begin{aligned}
x_{i t}^{\prime} & =t f-i d f\left(x_{i t}, \vec{x}_{i}\right) \\
& =\sqrt{t f\left(x_{i t}, \vec{x}_{i}\right)} \cdot \log \left(1+|V| / \sum_{j=1}^{|V|} t f\left(x_{i t}, \vec{x}_{j}\right)\right)
\end{aligned}
$$

The cosine similarity of attributes between nodes $v_{i}$ and $v_{j}$ is then defined as:

$$
\text { cosine }\left(\vec{x}_{i}^{\prime}, \vec{x}_{j}^{\prime}\right)=\vec{x}_{i}^{\prime} \cdot \vec{x}_{j}^{\prime} /\left(\left\|\vec{x}_{i}^{\prime}\right\| \cdot\left\|\vec{x}_{j}^{\prime}\right\|\right)
$$

Thereafter, we added weights to the aggregated graph. We calculated the proximity between a node and its direct neighbors in this new graph using:

$$
S=\lambda z e r o-\text { one }\left(S^{\text {topo }}\right)+(1-\lambda) \text { zero-one }\left(S^{\text {attr }}\right)
$$




\begin{tabular}{lcccc}
\hline Datasets & Nodes & Edges & Communities & Attributes \\
\hline Texas & 183 & 328 & 5 & 1,703 \\
Cornell & 195 & 304 & 5 & 1,703 \\
Washington & 217 & 446 & 5 & 1,703 \\
Wisconsin & 262 & 530 & 5 & 1,703 \\
Twitter & 171 & 796 & 7 & 578 \\
Cora & 2,708 & 5,429 & 7 & 1,433 \\
Citeseer & 3,312 & 4,732 & 6 & 3,703 \\
UAI2010 & 3,363 & 45,006 & 19 & 4,972 \\
Pubmed & 19,729 & 44,338 & 3 & 500 \\
\hline
\end{tabular}

Table 1: Datasets descriptions.

where $S^{\text {topo }}=\left[s_{i j}^{\text {topo }}\right]\left(s_{i j}^{\text {topo }}=\operatorname{cosine}\left(\vec{a}_{i}, \vec{a}_{j}\right)\right)$ and $S^{\text {attr }}=$ $\left[s_{i j}^{\text {attr }}\right]\left(s_{i j}^{\text {attr }}=\operatorname{cosine}\left(\vec{x}_{i}, \vec{x}_{j}\right)\right)$ are respectively the topological and attribute similarities between node $v_{i}$ and its direct neighbor $v_{j} \in \Gamma_{i}, \lambda$ is a tradeoff parameter, and zero-one $(\cdot)$ plays the role of normalization defined as:

$$
\text { zero-one }(\vec{y})=\left(y_{i}-\min (\vec{y})\right) /(\max (\vec{y})-\min (\vec{y}))
$$

Given the similarity matrix $S$ of the aggregated graph, we defined the pairwise constraint as:

$$
L_{r e g}=\sum_{i, j} s_{i j}\left\|\vec{z}_{i}-\vec{z}_{j}\right\|_{2}^{2}=2 \operatorname{tr}\left(Z^{T} \Psi Z\right)
$$

where $\Psi=F-S$ and $F$ is a diagonal matrix with $f_{i i}=$ $\sum_{j} s_{i j}$. We then incorporated the pairwise constraint as a graph regularization in the objective function to enhance community detection locally.

Finally, the objective of the model is to minimize the following loss function::

$$
L=\gamma L_{\text {topo }}+(1-\gamma) L_{a t t r}+\eta L_{r e g}
$$

where $\gamma$ and $\eta$ are the parameters that control the tradeoff between different parts of the loss. We used the backpropagation (BP) algorithm and Adam optimizer to train the model. At convergence, the algorithm produces the community label for each node by:

$$
\hat{c}_{i}=\underset{c_{i} \in L}{\arg \max } z_{i c_{i}}
$$

\section{Experiments}

We compared our approach with eight state-of-the-art methods on nine widely-used benchmark datasets. We also analyze the features of our method to appreciate its effectiveness.

\subsection{Experiment Setup}

To carry out an accurate comparison of the nine methods, we used nine public datasets with known communities (Table 1). As the networks used have ground-truth communities, we adopted two widely used metrics, i.e. accuracy (AC) [Liu et $a l ., 2012$ ] and normalized mutual information (NMI) [Danon et al., 2005], for performance evaluation. We applied Adam optimizer in our GUCD method using TensorFlow.

\subsection{Comparison with the Existing Methods}

We first evaluated our GUCD against eight (unsupervised) community detection methods. Depending on what network information they use, these existing methods can be grouped

\begin{tabular}{lccccccccc}
\hline \multirow{2}{*}{ Datasets } & \multicolumn{10}{c}{ AC (\%) } \\
\cline { 2 - 10 } & DCSBM & EdMot-SC & LDA & Block-LDA & PCLDC & SCI & MISAGA & TLSC & GUCD \\
\hline Texas & 48.09 & 48.09 & 56.28 & 54.10 & 38.80 & 62.30 & 46.99 & 65.02 & $\mathbf{6 7 . 7 6}$ \\
Cornell & 37.95 & 30.77 & 44.62 & 46.15 & 30.26 & 45.64 & 56.92 & 47.69 & $\mathbf{7 1 . 2 8}$ \\
Washington & 31.8 & 48.39 & 44.62 & 39.17 & 29.95 & 51.15 & 56.68 & 51.61 & $\mathbf{7 3 . 8 5}$ \\
Wsicsonsin & 32.82 & 32.06 & 44.62 & 49.62 & 30.15 & 50.38 & 66.79 & 49.23 & $\mathbf{7 6 . 7 2}$ \\
Twitter & 60.49 & 39.51 & 37.04 & 35.80 & 56.79 & 50.62 & 49.38 & $\mathbf{6 2 . 8 7}$ & $60.49(2)$ \\
Cora & 38.48 & 27.07 & 37.19 & 25.52 & 34.08 & 40.62 & 35.60 & 47.62 & $\mathbf{5 0 . 5 9}$ \\
Citeseer & 26.57 & 25.60 & 31.34 & 24.35 & 24.85 & 27.98 & 44.82 & 35.74 & $\mathbf{5 4 . 4 7}$ \\
UAI2010 & 2.60 & 18.42 & 34.07 & 16.04 & 28.82 & 30.94 & 15.81 & 29.28 & $\mathbf{3 7 . 5 8}$ \\
Pubmed & 53.64 & 39.29 & 46.30 & 49.01 & $\mathbf{6 3 . 5 5}$ & 47.39 & 42.35 & 61.38 & $63.13(2)$ \\
\hline AVG & 36.94 & 34.36 & 41.79 & 37.75 & 37.47 & 45.22 & 46.15 & 50.05 & $\mathbf{6 1 . 7 6}$ \\
\hline
\end{tabular}

Table 2: Comparison of the nine methods in AC. Bold font indicates the best result. The number in brackets is the rank of the algorithm.

\begin{tabular}{lccccccccl}
\hline Datasets & \multicolumn{10}{c}{ NMI (\%) } \\
\cline { 2 - 10 } & DCSBM & EdMot-SC & LDA & Block-LDA & PCLDC & SCI & MISAGA & TLSC & GUCD \\
\hline Texas & 16.65 & 18.79 & $\mathbf{3 1 . 2 9}$ & 4.21 & 10.37 & 17.84 & 26.56 & 23.92 & $29.65(2)$ \\
Cornell & 9.69 & 9.67 & 21.09 & 6.81 & 7.23 & 11.44 & 27.62 & 13.61 & $\mathbf{4 3 . 8 5}$ \\
Washington & 9.87 & 18.66 & 38.48 & 3.69 & 5.66 & 12.37 & 35.80 & 17.63 & $\mathbf{4 6 . 6 7}$ \\
Wsicsonsin & 3.14 & 11.28 & 46.56 & 10.09 & 5.01 & 17.03 & 37.06 & 16.65 & $\mathbf{4 7 . 5 6}$ \\
Twitter & 57.48 & 24.24 & 31.10 & 0.00 & 52.64 & 43.00 & 52.91 & 49.14 & $\mathbf{5 8 . 1 4}$ \\
Cora & 17.07 & 9.58 & 14.61 & 2.42 & 17.54 & 19.26 & 14.07 & $\mathbf{3 3 . 2 0}$ & $32.33(2)$ \\
Citeseer & 4.13 & 11.26 & 9.13 & 1.41 & 2.99 & 4.87 & 19.62 & 23.16 & $\mathbf{2 7 . 4 3}$ \\
UAI2010 & 31.21 & 12.58 & 35.42 & 5.70 & 26.92 & 24.80 & $\mathbf{3 8 . 5 4}$ & 22.87 & $33.35(3)$ \\
Pubmed & 12.28 & 0.21 & 10.55 & 6.58 & 26.84 & 5.59 & 10.79 & 19.63 & $\mathbf{2 6 . 9 8}$ \\
\hline AVG & 17.95 & 12.92 & 26.47 & 4.55 & 17.25 & 17.36 & 29.22 & 24.42 & $\mathbf{3 8 . 4 4}$ \\
\hline
\end{tabular}

Table 3: Comparison of the nine methods in NMI.

into three types. The first type uses only network topology, which includes DCSBM [Karrer and Newman, 2011] and EdMot-SC [Li et al., 2019]. The second uses only node attributes, including LDA [Blei et al., 2003]. The third uses topology and attributes together, including Block-LDA [Balasubramanyan and Cohen, 2011], PCLDC [Yang et al., 2009], SCI [Wang et al., 2016], MISAGA [He and Chan, 2018] and TLSC [Zhang et al., 2018].

GUCD is the best on 7 and 6 out of 9 datasets in terms of AC (Table 2) and NMI (Table 3). On the remaining networks where GUCD does not perform the best, it is still competitive with the best baselines. These results demonstrate the superiority of our new approach over the existing methods.

\subsection{Deep Analysis of GUCD}

Similar to most existing deep learning methods, GUCD has multiple components affecting its performance. Moreover, MRFasGCN is a major component of GUCD and can be applied in different ways. Here we report the results from different combinations of different major components of GUCD.

\section{Effects of Individual Components}

We compared GUCD with six variations of GUCD. We first considered two unsupervised variants of MRFasGCN, a major component of GUCD: 1) the weight parameters $H^{(0)}$, $H^{(1)}$ and $H^{(2)}$ in MRFasGCN is set to 1 without training (as the most general way), namely MasG-U1, and 2) the weight parameters is set randomly without training (as suggested as an unsupervised usage in [Kipf and Welling, 2017]), namely MasG-U2. We included in our comparison with another unsupervised variant of MRFasGCN by adding the inner product of node community membership for reconstructing links as the decoder, namely MasG-IN. (Note that this is the most common way used in unsupervised GCN models for network embedding, a different albeit similar problem.) We also tested three variants of GUCD. The first two reconstruct links and attributes separately, named as GUCD-1 and 2. The third is GUCD without the local enhancement, called GUCD-3.

The experimental results on nine benchmark problems revealed that GUCD performed the best on 7 and 7 out of 


\begin{tabular}{lcc|c|cccc|c}
\hline \multirow{2}{*}{ Datasets } & \multicolumn{7}{c}{ AC $\%$ ) } \\
\cline { 2 - 8 } & MasG-U1 & MasG-U2 & MasG-IN & GUCD-1 & GUCD-2 & GUCD-3 & GUCD \\
\hline Texas & 57.22 & 56.99 & 57.30 & 61.62 & $\mathbf{7 2 . 2 8}$ & 69.57 & $67.76(3)$ \\
Cornell & 43.72 & 43.43 & 44.16 & 47.47 & 70.26 & 67.18 & $\mathbf{7 1 . 2 8}$ \\
Washington & 48.42 & 48.42 & 63.76 & 66.36 & 71.43 & 72.02 & $\mathbf{7 3 . 8 5}$ \\
Wsicsonsin & 54.17 & 46.04 & 46.97 & 53.21 & 71.76 & 73.66 & $\mathbf{7 6 . 7 2}$ \\
Twitter & 55.30 & 31.00 & 51.85 & 50.00 & 55.56 & 56.79 & $\mathbf{6 0 . 4 9}$ \\
Cora & 34.96 & 32.50 & 36.40 & 37.27 & 49.17 & $\mathbf{5 1 . 2 9}$ & $\mathbf{5 0 . 5 9}(2)$ \\
Citeseer & 48.81 & 24.86 & 32.49 & 28.60 & 48.18 & 52.46 & $\mathbf{5 4 . 4 7}$ \\
UAI2010 & 16.25 & 16.72 & 16.34 & 15.96 & 34.00 & 36.12 & $\mathbf{3 7 . 5 8}$ \\
Pubmed & 39.98 & 39.95 & 50.84 & 51.41 & 62.09 & 61.33 & $\mathbf{6 3 . 1 3}$ \\
\hline AVG & 44.31 & 37.77 & 44.46 & 45.77 & 59.41 & 60.05 & $\mathbf{6 1 . 7 6}$ \\
\hline
\end{tabular}

Table 4: Comparison of GUCD with six variants (MasG-U1, MasGU2, MasG-IN, GUCD-1, GUCD-2 and GUCD-3) in AC.

\begin{tabular}{lcc|c|cccc|l}
\hline \multirow{2}{*}{ Datasets } & \multicolumn{7}{c}{ NMI (\%) } \\
\cline { 2 - 8 } & MasG-U1 & MasG-U2 & MasG-IN & GUCD-1 & GUCD-2 & GUCD-3 & GUCD \\
\hline Texas & 14.34 & 15.32 & 17.29 & 20.60 & $\mathbf{4 3 . 4 5}$ & 34.35 & $29.65(3)$ \\
Cornell & 9.33 & 9.05 & 6.80 & 9.22 & 41.65 & 38.10 & $\mathbf{4 3 . 8 5}$ \\
Washington & 10.35 & 10.35 & 24.25 & 28.07 & 46.16 & 37.59 & $\mathbf{4 6 . 6 7}$ \\
Wsicsonsin & 12.36 & 8.48 & 6.52 & 8.50 & 44.81 & 42.78 & $\mathbf{4 7 . 5 6}$ \\
Twitter & 38.02 & 21.36 & 48.60 & 35.18 & 55.36 & 47.60 & $\mathbf{5 8 . 1 4}$ \\
Cora & 13.03 & 7.08 & 12.43 & 19.38 & 27.31 & 29.76 & $\mathbf{3 2 . 3 3}$ \\
Citeseer & 22.43 & 5.74 & 12.07 & 9.08 & 21.40 & 27.03 & $\mathbf{2 7 . 4 3}$ \\
UAI2010 & 5.35 & 5.61 & 5.50 & 5.42 & 32.50 & $\mathbf{3 3 . 9 0}$ & $33.35(2)$ \\
Pubmed & 0.34 & 0.37 & 9.55 & 8.89 & 23.76 & 24.27 & $\mathbf{2 6 . 9 8}$ \\
\hline AVG & 13.95 & 9.26 & 15.89 & 16.04 & 37.38 & 35.04 & $\mathbf{3 8 . 4 4}$ \\
\hline
\end{tabular}

Table 5: Comparison of our GUCD with the six variants in NMI.

the 9 networks in terms of AC (Table 4) and NMI (Table 5). Specifically, GUCD is on average $17.45 \%$ (and $24.49 \%$ ) and $23.99 \%$ (and 29.18\%) more accurate than MasG-U1 and MasG-U2 in AC (and NMI). By some extra experiments we also observed that to achieve similar performances with GUCD, MRFasGCN needs almost 30\%, 30\%, 30\%, 30\%, $20 \%, 0.2 \%, 0.8 \%, 2 \%$ and $0.05 \%$ supervised information for the nine datasets respectively in training. This validates not only the effectiveness of our unsupervised framework using Autoencoder, but also the soundness of the communityoriented dual decoder, i.e. we use different while the most suitable mechanisms to reconstruct links and attributes respectively for community detection. Besides, GUCD is on average $17.3 \%$ (and 22.55\%) more accurate than MasG-IN in $\mathrm{AC}$ (and NMI). This further validates the soundness of the new decoding mechanisms (i.e. we reconstruct links by modelling that nodes in the same community share the same link pattern, and reconstruct attributes based on topic modelling) over the existing methods for reconstructing links alone via the inner product operation. In addition, GUCD is on average $15.99 \%$ (and $22.4 \%$ ), $2.35 \%$ (and $1.06 \%$ ), and $1.71 \%$ (and $3.4 \%$ ) more accurate than GUCD-1, GUCD-2, and GUCD-3 in terms of AC (and NMI). This demonstrates that the topological and attributed decoders both are effective. The strategy for local enhancement also helps meliorate overfitting caused by the sparsity of networked data.

\section{Qualitative Analysis}

To further validate the effectiveness of our new decoding mechanism over the one that most commonly used in network embedding, we further compared GUCD and MasGIN by showing the results of AC and NMI as a function of the number of iterations. Consider the results on Twitter as an example. As shown in Fig. 2, the GUCD results match more closely to the ground truth than MasG-IN, and the result of the former is more stable than that of the latter with more training cycles, suggesting that the new decoding mechanisms are suitable for unsupervised community detection.
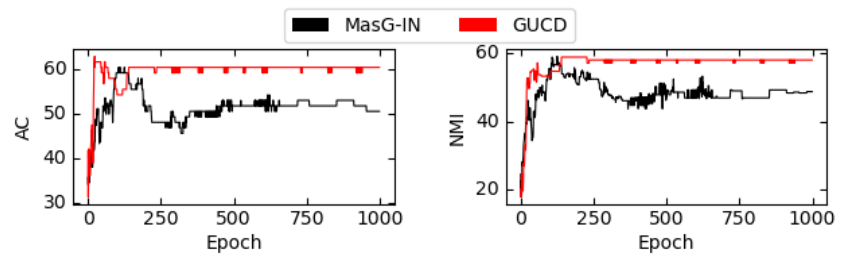

Figure 2: AC and NMI values as a function of iterations on Twitter.

\section{Related Work}

The current work of GCN focuses primarily on node classification [Kipf and Welling, 2017], which has been adopted for semi-supervised community detection [Li et al., 2018; Jin et al., 2019]. However, community detection is, in essence, an unsupervised problem since little training data are available for most applications. It is imperative to develop novel GCN methods for unsupervised community finding.

A related line of work is network embedding using GCN. For example, the ARGA method [Pan et al., 2018] adopts an adversarially regularized graph Autoencoder, which uses the same encoder and decoder as GAE [Kipf and Welling, 2016] while introduces an adversarial module to force the node representation to follow a suitable prior distribution. The ARGA method has also been extended to use graph convolution, instead of inner product, as the decoder to reconstruct links; the resulting method can reconstruct links better than reconstructing both links and attributes [Pan et al., 2019]. For unsupervised learning, most of the existing methods focus primarily on reconstructing network structures, as their performance degrades when being used to reconstruct links and attributes together. These existing methods seem to be suitable for representation learning, e.g., embedding, but not adequate for unsupervised community detection, a more difficult learning task. This has been experimentally shown by the comparison between GUCD and its variant MasG-IN (using inner product to reconstruct links), as shown in Fig. 2 and Tables $4 \& 5$.

\section{Conclusion and Discussion}

This is the first approach extending GCN effectively to unsupervised community detection. Using the Autoencoder framework, we focused on community identification rather than network embedding in the encoder; and used the most suitable mechanisms to reconstruct links and attributes separately. We also introduced a local enhancement to ameliorate the issue of overfitting brought by the sparsity of networked data. We carried out experiments and demonstrated the superiority of the new approach. We further showed the effectiveness of the novel decoding mechanism for generating links and attributes against the most commonly used methods for reconstructing links alone. Even though the new approach was designed for community detection, the underlying idea may be readily extended to GCN-based network embedding as discussed in Related Work.

\section{Acknowledgments}

This work was supported by the Natural Science Foundation of China (61832014, 61876128, 61772361, U1736103). 


\section{References}

[Balasubramanyan and Cohen, 2011] Ramnath Balasubramanyan and William W. Cohen. Block-lda: Jointly modeling entity-annotated text and entity-entity links. In Proceedings of the Eleventh SIAM International Conference on Data Mining, 2011.

[Blei et al., 2003] David M. Blei, Andrew Y. Ng, Michael I. Jordan, and John Lafferty. Latent dirichlet allocation. Journal of Machine Learning Research, 3:993-1022, 2003.

[Chen et al., 2018] Zhengdao Chen, Xiang Li, and Joan Bruna. Supervised community detection with line graph neural networks. arXiv preprint arXiv:1705.08415, 2018.

[Danon et al., 2005] Leon Danon, Albert Díaz-Guilera, Jordi Duch, and Alex Arenas. Comparing community structure identification. Journal of Statistical Mechanics: Theory and Experiment, 2005(09):P09008-P09008, sep 2005.

[Falih et al., 2018] Issam Falih, Nistor Grozavu, Rushed Kanawati, and Younès Bennani. Community detection in attributed network. In Companion Proceedings of the The Web Conference 2018, pages 1299-1306, 2018.

[Fortunato and Hric, 2016] Santo Fortunato and Darko Hric. Community detection in networks: A user guide. Physics Reports, 659:1-44, 072016.

[Fortunato, 2010] Santo Fortunato. Community detection in graphs. Physics Reports, 486:75 - 174, 2010.

[He and Chan, 2018] T. He and K. C. C. Chan. Misaga: An algorithm for mining interesting subgraphs in attributed graphs. IEEE Transactions on Cybernetics, 48(5):13691382, May 2018.

[Jin et al., 2018] Di Jin, Meng Ge, Liang Yang, Dongxiao He, Longbiao Wang, and Weixiong Zhang. Integrative network embedding via deep joint reconstruction. In Proceedings of the 27th International Joint Conference on Artificial Intelligence, pages 3407-3413, 72018.

[Jin et al., 2019] Di Jin, Ziyang Liu, Weihao Li, Dongxiao $\mathrm{He}$, and Weixiong Zhang. Graph convolutional networks meet markov random fields: Semi-supervised community detection in attribute networks. In Proceedings of the 33rd AAAI Conference on Artificial Intelligence, 2019.

[Karrer and Newman, 2011] Brian Karrer and M. E. J. Newman. Stochastic blockmodels and community structure in networks. Physical Review E, 83:016107, Jan 2011.

[Kingma and $\mathrm{Ba}, 2015]$ Diederik P. Kingma and Jimmy Ba. Adam: A method for stochastic optimization. In Proceedings of the 3rd International Conference on Learning Representations, 2015.

[Kipf and Welling, 2016] Thomas N. Kipf and Max Welling. Variational graph auto-encoders. CoRR, abs/1611.07308, 2016.

[Kipf and Welling, 2017] Thomas N. Kipf and Max Welling. Semi-supervised classification with graph convolutional networks. In Proceedings of the 5th International Conference on Learning Representations, 2017.
[Li et al., 2018] Qimai Li, Zhichao Han, and Xiao-Ming Wu. Deeper insights into graph convolutional networks for semi-supervised learning. In Proceedings of the 32nd AAAI Conference on Artificial Intelligence, pages 35383545, 2018.

[Li et al., 2019] Pei-Zhen Li, Ling Huang, Chang-Dong Wang, and Jian-Huang Lai. Edmot: An edge enhancement approach for motif-aware community detection. In Proceedings of the 25th ACM SIGKDD International Conference on Knowledge Discovery and Data Mining, page 479-487, 2019.

[Liu et al., 2012] Haifeng Liu, Zhaohui Wu, Deng Cai, and Thomas S. Huang. Constrained nonnegative matrix factorization for image representation. IEEE Transactions on Pattern Analysis and Machine Intelligence, 34(7):12991311, July 2012.

[Monti et al., 2018] Federico Monti, Karl Otness, and Michael M. Bronstein. MOTIFNET: A motif-based graph convolutional network for directed graphs. In 2018 IEEE Data Science Workshop, pages 225-228, 2018.

[Pan et al., 2018] Shirui Pan, Ruiqi Hu, Guodong Long, Jing Jiang, Lina Yao, and Chengqi Zhang. Adversarially regularized graph autoencoder for graph embedding. In Proceedings of the 27th International Joint Conference on Artificial Intelligence, pages 2609-2615, 2018.

[Pan et al., 2019] Shirui Pan, Ruiqi Hu, Sai fu Fung, Guodong Long, Jing Jiang, and Chengqi Zhang. Learning graph embedding with adversarial training methods. arXiv preprint arXiv:1901.01250, 2019.

[Wang et al., 2016] Xiao Wang, Di Jin, Xiaochun Cao, Liang Yang, and Weixiong Zhang. Semantic community identification in large attribute networks. In Proceedings of the 30th AAAI Conference on Artificial Intelligence, 2016.

[Wang et al., 2017] Xiao Wang, Peng Cui, Jing Wang, Jian Pei, Wenwu Zhu, and Shiqiang Yang. Community preserving network embedding. In Proceedings of the 31st AAAI Conference on Artificial Intelligence, 2017.

[Yang et al., 2009] Tianbao Yang, Rong Jin, Yun Chi, and Shenghuo Zhu. Combining link and content for community detection: A discriminative approach. In Proceedings of the 15th ACM SIGKDD International Conference on Knowledge Discovery and Data Mining, pages 927-936. ACM, 2009.

[Yang et al., 2016] Liang Yang, Xiaochun Cao, Dongxiao He, Chuan Wang, Xiao Wang, and Weixiong Zhang. Modularity based community detection with deep learning. In Proceedings of the 25th International Joint Conference on Artificial Intelligence, pages 2252-2258, 2016.

[Zhang et al., 2018] Ge Zhang, Di Jin, Jian Gao, Pengfei Jiao, Françoise Fogelman-Soulié, and Xin Huang. Finding communities with hierarchical semantics by distinguishing general and specialized topics. In Proceedings of the 27th International Joint Conference on Artificial Intelligence, pages 3648-3654, 2018. 